(mean age at surgery: 1.4 years) and 32 healthy subjects. ROCFT was scored according to three different validated scoring methods.

Results Results varied markedly between the scoring methods. When scored according to Meyers \& Meyers, patients performed significantly worse than controls in the copy task ( $\mathrm{p}=0.03$ ), whereas no significant differences were found in the memory task. Scoring according to Wallon and Mesmin showed clear differences between subjects and controls with respect to the approach how to construct the figure: $78 \%$ of CHD patients (controls: $47 \%$ ) displayed a unstructured or intermediate approach to drawing the figure, whereas only $22 \%$ of CHD patients (controls: $53 \%$ ) chose a complex approach $(\mathrm{p}=0.001)$. Scoring according to Bernstein et al. showed no differences between groups.

Conclusions Adolescents with surgically treated CHD demonstrate deficits in visuospatial and executive function. The ROCFT provides information on different functional aspects, which cannot adequately be assessed with one single scoring method.

\section{BORN TOO SMALL OR TOO EARLY - THE EFFECT OF PHYSICAL FITNESS IN YOUNG ADULTHOOD ON COGNITIVE PERFORMANCE}

doi:10.1136/archdischild-2012-302724.0154

J Svedenkrans, E Henckel, J Kowalski, K Bohlin, M Norman. Department of Clinical Science, Intervention and Technology, Karolinska Institute, Stockholm, Sweden

Background and aims Physical exercise has been demonstrated to give positive cognitive effects. We have previously reported lower exercise capacity in otherwise healthy young men with low birth weight or preterm birth. The objective of this study was to investigate the association between perinatal risk factors, cognitive performance and physical fitness in young adulthood.

Methods This was a population-based cohort study including 218,915 young men born in Sweden 1973-1981, conscripted for military service in 1993-2001. Data on birth characteristics was obtained from the Medical Birth Register and linked to information on cognitive test scores and results on ergometer cycling test from the Military Conscript Register.

Results Exercise capacity, as measured by ergometer cycling, was positively associated with cognitive performance, as measured by global intelligence stanine scores, $p<0.001$. The subgroup with shortest gestational age, less than 28 weeks, exhibited both the lowest cognitive test scores and the lowest exercise capacity. Low birth weight standard deviation scores (BWSDS) for gestational age was associated with lower cognitive performance in a stepwise manner. Using ordinal regression analysis, low BWSDS remained independently associated with low cognitive scores in young adult age.

Conclusions The lower cognitive performance observed in young adults born prematurely or with low birth weight is associated with physical fitness. Targeting physical exercise may be an intervention to enhance cognitive performance and educational achievements in a population at risk, such as survivors of preterm birth and poor fetal growth.

\section{EFFECT OF IN UTERO EXPOSURE TO ISCHEMIC-HYPOXIC CONDITIONS ON CHILDHOOD ATTENTION DEFICIT HYPERACTIVITY DISORDER}

doi:10.1136/archdischild-2012-302724.0155

1,2D Getahun, ${ }^{3} \mathrm{GG}$ Rhoads, ${ }^{3} \mathrm{~K}$ Demissie, ${ }^{4} \mathrm{~L}$ Shou-En, ${ }^{1} \mathrm{VP}$ Quinn, ${ }^{5} \mathrm{MJ}$ Fassett, ${ }^{6} \mathrm{DA}$ Wing, 'SJ Jacobsen. 'Research \& Evaluation, Kaiser Permanente Southern California Medical Group, Pasadena, CA; ${ }^{2}$ Obstetrics \& Gynecology, UMDNJ - Robert Wood Johnson Medical School, New Brunswick; ${ }^{3}$ Epidemiology; ${ }^{4}$ Biostatistics, UMDNJ - School of Public Health, Piscataway, NJ; ${ }^{5}$ Maternal-Fetal Medicine, Kaiser Permanente Southern California Medical Group, Pasadena; ${ }^{6}$ Obstetrics \& Gynecology, University of California, Irvine, Irvine, CA, USA
Objective To examine the association between ischemic-hypoxic conditions (IHC) and Attention Deficit Hyperactivity Disorder (ADHD) by gestational age at delivery and race/ethnicity.

Methods A nested case-control study using the Kaiser Permanente Southern California (KPSC) medical records. Study cohort were children aged 5-11 years who were delivered and cared for in KPSC Healthcare system between 1995-2010 ( $\mathrm{n}=308,634)$. Cases were children with a clinical diagnosis of $\mathrm{ADHD}$ and obtained at least 2 prescriptions specific to ADHD during the follow-up period. For each case, five controls matched to cases on child age at time of diagnosis were selected. Exposures were defined based on ICD-9 codes. A conditional logistic regression model was used to estimate adjusted odds ratios (OR).

Results Among eligible children, 13,613 (4.3\%) had a diagnosis of ADHD. Compared to control children, case children were more likely to be male and of White or African-American race/ethnicity. Case children than controls were more likely to be exposed to IHC $(\mathrm{OR}=1.16,95 \%$ confidence intervals [CI] 1.11-1.21). Analysis of cases and controls stratified by gestational age revealed that case children born at 28-33, 34-36, and 37-42 weeks of gestation, were significantly more likely to be exposed to IHC; 1.6 -fold $(95 \%$ CI, 1.2-2.2), 1.2-fold (95\% CI, 1.0-1.4), and 1.1-fold (95\% CI, 1.0-1.2), respectively, compared to control children. IHC was associated with increased odds of $\mathrm{ADHD}$ across all race/ethnicity groups.

Conclusion These findings suggest that IHC is independently associated with an increased risk of childhood $\mathrm{ADHD}$ especially in early preterm birth.

\section{CEFAZOLIN PLASMA PROTEIN BINDING AND ITS COVARIATES IN NEONATES}

doi:10.1136/archdischild-2012-302724.0156

${ }^{1} \mathrm{~A}$ Smits, ${ }^{2,3} \mathrm{~A}$ Kulo, ${ }^{2} \mathrm{R}$ Verbesselt, ${ }^{1} \mathrm{~V}$ Cossey, ${ }^{2} \mathrm{~J}$ de Hoon, ${ }^{4} \mathrm{P}$ Vermeersch, ${ }^{1} \mathrm{~K}$ Allegaert. ${ }^{1}$ Neonatal Intensive Care Unit; ${ }^{2}$ Center for Clinical Pharmacolgoy, University Hospitals

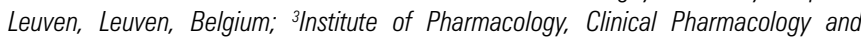
Toxicology, Faculty of Medicine, University of Sarajevo, Sarajevo, Bosnia-Herzegovina; ${ }^{4}$ Department of Laboratory Medicine, University Hospitals Leuven, Leuven, Belgium

Background and aim Cefazolin (CFZ) is highly and saturably bound to albumin in adults. It is mainly used as prophylactic antibiotic agent. The aim of the present study is to describe CFZ protein binding and its covariates in neonates.

Methods Neonates to whom intravenous CFZ (50 mg/kg) was administered as standard care prior to an invasive procedure were included. Total and unbound CFZ plasma concentrations were determined at $1 / 2 ; 2 ; 4$ and 8 hours after CFZ administration. Linear and multiple regression analyses were used to document covariates of unbound CFZ fraction. For paired analysis of unbound CFZ fractions Wilcoxon signed rank test was used.

Results Forty patients with median weight 2767 (range 830-4200) grams and median postmenstrual age (PMA) 39 (25-45) weeks were included. Median unbound CFZ fraction was 0.39 (0.10-0.73). Linear regression of unbound CFZ fraction versus unbound CFZ plasma concentration $\left(R^{2}=0.39\right)$ had a slope significantly different from zero $(p<0.001)$. In a multiple regression analysis, albuminaemia, total CFZ concentration, indirect bilirubinaemia and PMA resulted in an $R^{2}$ value of 0.496 . Median unbound CFZ fraction at peak concentration $(0.46$; range $0.28-0.69)$ was significantly higher compared to trough level (0.36; range 0.17-0.73) $(p<0.001)$.

Conclusions Between patient and within patient saturability of CFZ protein binding were also documented in neonates. We revealed a median plasma unbound CFZ fraction of 0.39 in neonates, which is higher than reported values in adults. The integration of CFZ protein binding aspects in future pharmacokinetic/pharmacodynamic research is warranted to optimize CFZ dosing, especially in neonates. 
157 SURVEY ON USE OF CAFFEINE IN APNOEA OF PREMATURITY IN NEONATAL UNITS ACROSS ENGLAND

doi:10.1136/archdischild-2012-302724.0157

D Abraham, P Rajagopal, E Curtis, A Manzoor, M Ahmed. Paediatrics, Queens Hospital, Burton upon Trent, UK

Background Apnoea of prematurity (AOP) is a significant clinical problem in premature infants and is almost universal in infants $<1000 \mathrm{~g}$ at birth. Caffeine has emerged as the methylxanthine of choice to treat AOP. Although it is commonly used, there is no unified consensus or guideline on its use in NNUs in England.

Aim To study the current practice of caffeine use in AOP at NNUs in England.

Methods A telephonic survey of level 3 and level 2 units in England was conducted, using a standardised questionnaire, over November and December, 2011.

Results Out of 52 units surveyed, 48\% were level 3 units. All units used caffeine for treatment of AOP (base $60 \%$ and citrate $40 \%$ of units). $92 \%$ of units have written guidelines on caffeine use. Caffeine was started by $47 \%$ of units based on gestational age, regardless of symptoms.[IS3] All units used a loading dose, which varied between 5 and $25 \mathrm{mg} / \mathrm{kg}$ (median of $10 \mathrm{mg} / \mathrm{kg}$ ) for caffeine base and 15 to $20 \mathrm{mg} / \mathrm{kg}$ (median of $20 \mathrm{mg} / \mathrm{kg}$ ) for citrate. The maintenance dose varied between $2.5-6 \mathrm{mg} / \mathrm{kg} /$ day (median of $5 \mathrm{mg} / \mathrm{kg} /$ day) for base and $5-12 \mathrm{mg} / \mathrm{kg} /$ day (median of $5 \mathrm{mg} / \mathrm{kg} /$ day) for citrate. Caffeine levels were routinely performed by $7 \%$ of units. Caffeine was discontinued between 30 to 36 weeks gestation.

Discussion Our survey depicts that practice of caffeine use varies significantly across NNUs in England. The results from this survey could be used as a footing for further data collection, for formulation of a uniform guideline maximising the utilisation of this extensively studied drug.

\section{A SYSTEMATIC REVIEW OF PHARNMACOKINETICS (PK) OF DOBUTAMINE FOR USE IN NEONATES AND CHILDREN}

doi:10.1136/archdischild-2012-302724.0158

${ }^{1}$ GS Shah, ${ }^{2} \mathrm{D}$ Crook, ${ }^{3} \mathrm{H}{ }^{4}$ Rojas-Anaya, H Rabe. 'Paediatric Academic Department, Brighton and Sussex Medical School; ${ }^{2}$ Clinical Investigations and Research Unit, ${ }^{3}$ Paediatric Academic Department, Brighton and Sussex University Hospitals; ${ }^{4}$ Paediatric Academic Department, Brighton and Sussex University Hospitals, Brighton and Sussex Medical School, Brighton, UK

Background Dobutamine has been used off-label in newborns and children for treating haemodynamic insufficiency for over 20 years. As preparation for a large randomised study to achieve Paediatric Use Marketing Authorisation for dobutamine in newborns we performed a structured literature review of PK data.

Methods Structured searches were conducted using the electronic databases Medline and Embase. Search terms included dobutamine, infant, newborn, paediatric/pediatric, prematurity, child, infant, low birth weight infant, preschool child, school child, adolescent, pharmacokinetics, clinical pharmacology. Data was extracted based on pre-defined criteria decided by the team.

Main results Six of eleven papers emerging from this search met our inclusion criteria. These reported dobutamine PK data in a combined total of 70 children (age range 0 days-22 years, 13 newborns, 27-42 weeks gestation), with infusion rates ranging from $0.5-20$ $\mu \mathrm{g} / \mathrm{kg} / \mathrm{min}$. Five papers found that the infusion rate was positively correlated to plasma dobutamine concentration. Dobutamine clearance rates showed great variability between individuals (range 35.1$482.2 \mathrm{~mL} / \mathrm{kg} / \mathrm{min}$ ). Four papers found that clearance did not vary with infusion rate, suggesting first order kinetics, although one paper $(n=12)$ showed a significantly negative relationship $(p<0.001)$ of dobutamine clearance to steady-state plasma concentration (dobutamine dosage $2-15 \mu \mathrm{g} / \mathrm{kg} / \mathrm{min}$ ).
Conclusions The current dobutamine PK data is difficult to interpret due to inhomogeneity and variability of patients' age and conditions, dobutamine dosages and study designs. High quality prospective PK data -especially in newborns- is urgently required prior to our large randomised study.

\section{PHARMACOKINETICS (PKS) OF LEVOSIMENDAN (LEVO) AND INTERMEDIATE METABOLITES (OR-1855 AND OR- 1896) IN NEWBORNS UNDERGOING CARDIOVASCULAR SURGERY WITH CARDIOPULMONARY BYPASS (CPB)}

doi:10.1136/archdischild-2012-302724.0159

${ }^{1} \mathrm{~A}$ Pellicer, ${ }^{2} \mathrm{R}$ Lubomirov, 'MC Bravo, 'P López-Ortego, ' $\mathrm{J}$ Pérez-Rodríguez, '1 Quero, ${ }^{1} \mathrm{~F}$ Cabañas. 'La Paz University Hospital, Department of Neonatology; ${ }^{2}$ Department of Clinical Pharmacology, La Paz University Hospital and Clinical Pharmacology Center, School of Medicine, Universidad Autónoma de Madrid. IdiPAZ., Madrid, Spain

Background and aims LEVO is a novel inodilator developed to treat heart failure. Biotransformation of LEVO in the intestinal tract gives rise to intermediate metabolites with prolonged beneficial haemodynamic effects. There are no data on LEVO PKs in neonates. We aim to investigate LEVO and intermediate metabolites PKs in newborns undergoing CPB.

Methods Eleven infants received step-wise dose increase of LEVO $(0.10,0.15,0.20 \mathrm{mg} / \mathrm{k} / \mathrm{min})$ delivered as i.v. continuous infusion, starting before CPB up to $48 \mathrm{~h}$ post-surgery. Eleven blood samples per subject were collected up to day 14 post-infusion started. Samples were quantified by HPLC-MS/MS. Non-compartmental methods were used for PK parameters. Median (IOR) values are reported. Results Area under the curve (AUC, ng*h/mL) OR-1855 plasma concentration [1717.10 (930.38-3756.41)] was 2.3- and 8.2-fold higher than LEVO [742.10 (527.23-1046)] and OR-1896 [209.78 (99.54-275.36)], respectively. LEVO clearance (CL, L/h/k) was 0.67 (0.44-1.0). OR-1855 maximum concentration (Cmax, $\mathrm{ng} / \mathrm{ml}$ ) was 5.2-fold higher than OR-1896 [18.5 (10.44-33.25) vs. 3.58 (2.94-4.38)]. OR-1896 and OR-1855 Cmax were respectively achieved $2 \mathrm{~h}$ before and $120 \mathrm{~h}$ after LEVO infusion stopped. LEVO CL increased and AUC decreased with postnatal age, explaining $66.23 \%$ $(p=0.023)$ and $34.51 \%(p=0.047)$ of their respective variance. LEVO AUC and pre-surgery antibiotics explained $38.89 \%(\mathrm{p}=0.016)$ and $26.68 \%(p=0.035)$ of OR-1855 AUC variance, respectively. Use of additional diuretics to furosemide explained $27.21 \%(\mathrm{p}=0.025)$ of OR-1896 AUC. No other covariates influenced LEVO or metabolites PKs.

Conclusions This study describes the pharmacokinetic profile of LEVO and intermediate metabolites in newborns as well as covariates explaining a significant part of their variance.

\section{LONGTERM OUTCOME AFTER LIVER TRANSPLANTATION: PSYCHOSOCIAL AND COGNITIVE ISSUES}

doi:10.1136/archdischild-2012-302724.0160

KH Schulz. University Medical Center Hamburg-Eppendorf, Hamburg, Germany

Psychosocial outcome in liver transplanted children was primarily investigated in terms of health related quality of life (HRQOL). In children this multidimensional construct additionally accentuates domains like school, family, and peers as well as physical and cognitive-emotional development.

Although organ transplantation is lifesaving, recipients trade a terminal illness for a chronic syndrome with good organ function in most cases, however. Nevertheless, restoration of organ function does not involve return to a normal life. It is characterized by fear of organ failure and complications, side effects of the medication, developmental deficits, and psychiatric comorbidities. Liver transplanted children show a poorer HRQOL compared with the healthy population, equal to or better than in children with other chronic diseases. Factors 